\title{
Prioritising the Replanting Schedule of Seedling Tea Fields on Tea Estates for Drought Susceptibility Measured by the SWAPDT Method in the Absence of Historical In-filling Records
}

\author{
Christopher Nyarukowa ${ }^{1}$, Robert Koech ${ }^{1,2}$, Theodor Loots ${ }^{3}$, Jos Hageman $^{4} \&$ Zeno Apostolides ${ }^{1}$ \\ ${ }^{1}$ Department of Biochemistry, University of Pretoria, Hatfield, South Africa \\ ${ }^{2}$ Kenya Agriculture and Livestock Research Organization, Tea Research Institute, Kericho, Kenya \\ ${ }^{3}$ Department of Statistics, University of Pretoria, Hatfield, South Africa \\ ${ }^{4}$ Department of Mathematical and Statistical Methods, Wageningen University and Research, Wageningen, \\ Netherlands \\ Correspondence: Zeno Apostolides, Department of Biochemistry, University of Pretoria, Private Bag X20, \\ Hatfield 0028, South Africa. Tel: 27(0)12-420-2486. Fax: 27(0)12-362-5302. E-mail: zeno.apostolides@up.ac.za
}

Received: April 19, 2018

doi:10.5539/jas.v10n7p26

\author{
Accepted: May 19, $2018 \quad$ Online Published: June 15, 2018 \\ URL: https://doi.org/10.5539/jas.v10n7p26
}

\begin{abstract}
Due to the unpredictable natural droughts that occur, causing tea farmers significant losses in tea estates, a two-day method for distinguishing between drought tolerant (DT) and drought susceptible (DS) Camellia sinensis cultivars was developed. This work was based on known cultivars developed at the Tea Research Institute in Kenya and the Tea Research Foundation for Central Africa in Malawi. This paper contains an in-depth description of the application of the SWAPDT method on four 60-year old, C. sinensis seedling fields in Kenya. The in-filling history of the four fields due to drought-related deaths was obtained from historical records. The SWAPDT method scores correlated very well with the historical records. It has been indicated, from the results obtained in this study, that a sample size of 20 tea trees is sufficient to accurately determine the drought susceptibility of a large tea field of approximately 5-20 hectares, containing 50 000-200 000 tea trees, were the difference between their mean values, as measured by the SWAPDT method, is approximately $10 \%$.
\end{abstract}

Keywords: Camellia sinensis, drought tolerance, field comparison, relative water content, SWAPDT, tea estate

\section{Introduction}

Tea made from the leaves of Camellia sinensis, as green or black tea, has been drunk as a mild stimulant due to the caffeine content, since time immemorial (Ellis \& Nyirenda, 1995). Tea consumption has increased in recent years, due to the health-promoting effects associated with its high polyphenol content (Preedy, 2012). C. sinensis is cultivated in over 52 countries around the world. Global world trade is approximately $78 \%$ by value in the form of black, $20 \%$ as green and $2 \%$ as oolong tea (Nyarukowa, Koech, Loots, \& Apostolides, 2016). It is an important cash crop for countries such as India and China; in Africa alone, several countries produce tea, namely in Kenya, which is currently ranked third behind Sri Lanka and India with regards to annual production and export of black tea (FAO, 2015), Malawi, Uganda, Tanzania, Zimbabwe, Rwanda, South Africa, Burundi and Mauritius. C. sinensis tea estates need to be replanted every 20-90 years to maintain high yields. Tea estates are planted in sample blocks of about 5-20 hectares, at 10,000 trees per hectare, with seeds from the same batch. Most tea estates in Africa are planted with tea seeds procured from tea-baries (orchards) in India or Sri Lanka. The seed selection criteria employed focused on yield and neglected to consider drought tolerance (Murakami, Nakamura, Matsuda, \& Yoshikawa, 1999). During severe natural droughts, some of the trees (5-15\%) die and are replaced with new trees. Tea planters refer to this process as "in-filling." Most estates keep good records of the in-filling, and hence good and poor fields are easily identified. However, sometimes these records are missing, and a new method is required to determine the drought tolerance of a tea field that might be 20-90 years old (Willson \& Clifford, 2012).

Tea producers demand new cultivars which are DT, to reduce crop losses. In the coffee industry, farmers are faced with the problem of dealing with coffee rust. How they deal with this is by assessing and analysing the risk 
of an epidemic by considering the region's characteristics such as climate, soils, crop management patterns, namely shade management, etc. (DaMatta, 2004). This assessment approach has been adopted from studies conducted in West Africa on groundnuts (Avelino, Willocquet, \& Savary, 2004) as well as on work conducted by (Savary et al., 2000) on tropical Asian rice. As a result of this, tea farmers are also looking for an inexpensive yet effective method of determining which sample blocks of tea have a high percentage of DS plants so that these sample blocks may be prioritized for replanting. The samples in this study were collected from the James Finlay's estate in Kericho, Kenya which together with surrounding estates (Figure 1) produces 23 million kilograms of tea annually. This part of Kenya enjoys deep rich loam soils, which are high in organic content and combined with the perfect climate and environment are ideal for high yields of good quality tea (http://www.finlays.net). In Nyarukowa et al. (2016), a novel logistics probability formula was developed, which can be used to calculate a new cultivar's probability to be DT after employing the Short-time Withering Assessment of Probability for Drought Tolerance (SWAPDT) method. The aim of this study was to determine how many tea trees are needed per field to obtain a representative sample of the tea field so that tea fields can be prioritised for replanting.
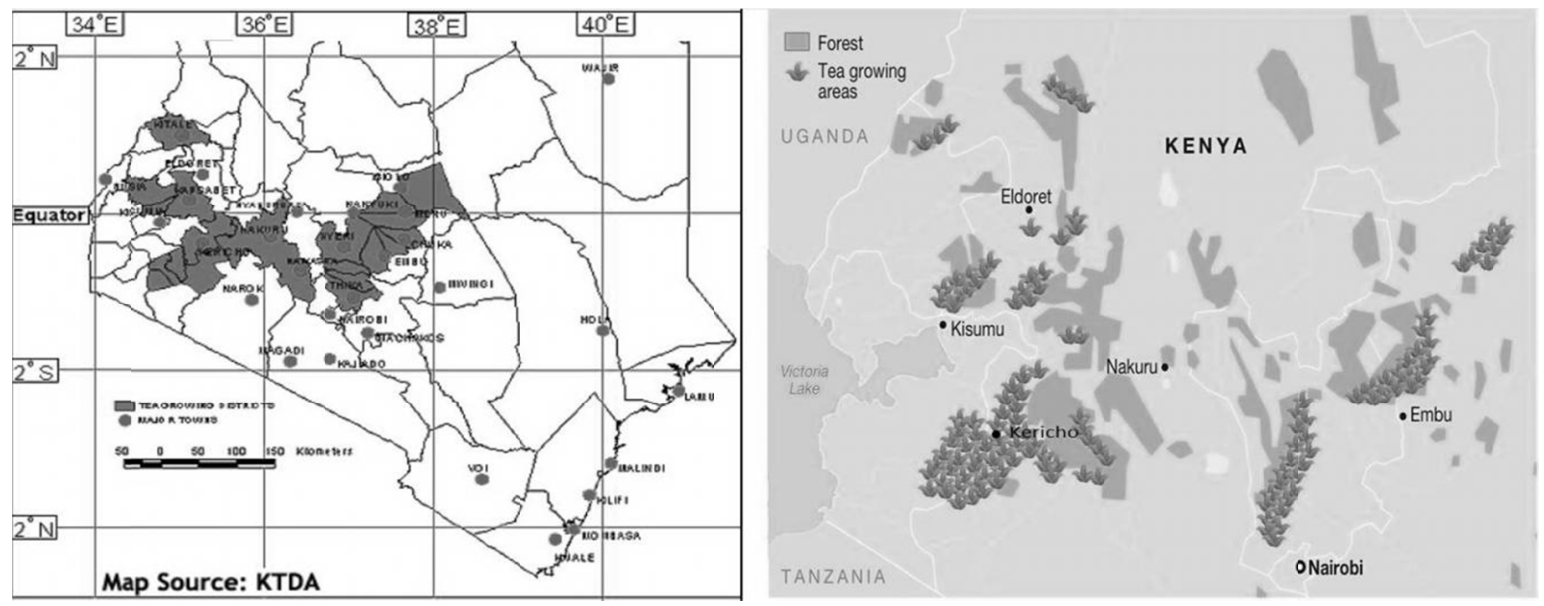

Figure 1. Tea growing areas in Kenya

Source: Kenya Tea Development Agency (http://blog.dominiontea.com/2014/03/27/kenyan-tea-industry).

\section{Statistical Analysis}

Statistical approaches for the analysis of metabolomic data vary. The first step involves the analysis/focus on single trait of interest (univariate analysis), or the effects of multiple metabolites on the outcome being studied (multivariate analysis). Results can then be validated using different approaches and fed into the multivariate analysis. Spatial regression models have been widely employed in biological science disciplines. Central to spatial analysis and quantitative geography, has been the study of methods that specify and fit spatial regression models (Bivand, Hauke, \& Kossowski, 2013). The most popular spatial correlation test is based on the Moran I test statistic. This test is in a normalised quadratic form, with regards to the variables being tested for spatial correlation (Kelejian \& Prucha, 2010). The Moran I test statistic functions to test that the spatial autocorrelation of a variable in the null hypothesis is zero. Upon the rejection of the null hypothesis, the variable will be deemed to be spatially auto correlated (Ord \& Getis, 1995). The Monte Carlo test can also be employed when dealing with a null hypothesis, $\mathrm{H}_{\mathrm{o}}$, and a corresponding dataset, in which the value $\mathrm{u}_{1}$ of a selected test statistic, $\mathrm{u}$, is ranked amongst a set of analogous values generated by randomly sampling from the null distribution of $u$. The rank of the test statistic $u_{1}$, when the $u$ distribution is continuous amongst the set of values $\left\{u_{i}: i=1, \ldots m\right\}$ enables the determination of the exact significance level for the test (Besag \& Diggle, 1977). The ordinary linear regression (OLR) model is amongst the most useful statistical methods employed in spatial analysis to identify relationships between variables is (Mei, He, \& Fang, 2004). In this technique, the dependent variable, $y$, is modelled as a linear function of a set of independent variables $x_{1}, x_{2}, \ldots x_{\mathrm{p}}$. Based on $\mathrm{n}$ observations $\left(y i ; x i_{1}\right.$, $\left.x i_{2}, \ldots x i_{\mathrm{p}}\right),(\mathrm{i}=1,2, \ldots \mathrm{n})$, from a study region, the model can be expressed as:

$$
y_{i}=\beta_{0}+\sum_{k=1}^{\rho} \beta_{k} x_{i k}+\epsilon_{i}
$$


where, $\beta_{0}, \beta_{1}, \ldots \beta_{p}$ are parameters, and $\epsilon_{1}, \epsilon_{2}, \ldots \epsilon_{n}$ are error terms assumed to be normally distributed, random, independent variables (Mei et al., 2004).

Determining a study's sample size requires a compromise, which balances power, economy, and timeliness (Dupont \& Plummer, 1990). Researchers must define the sample size, power, and hypotheses for their study and to effectively do this, they require software programs that can calculate the missing parameter when given any two of the preceding three parameters. These programs compute the sample size required to identify, using a specified power, e.g., the difference in efficacy of a particular treatment, the power this difference is detectable with given sample size, and the detectable difference with particular power and sample size. The link between the power and its corresponding sample size is best seen by plotting the power curve as a function of the parameter of interest (Kelsey et al., 1986).

The Student's t-test was used to calculate the probability (p) that the two samples belong to the same population. When $\mathrm{p}<0.05$, there is a $95 \%$ certainty that the two samples belong to different populations. The Standard Error of the Mean (SEM) is an inferential statistic that can be used to draw error bars on histograms to visually estimate the p-value. When the sample size (n) $>10$, and the gap between the SEM error bars $>$ SEM1 + SEM2, we can be $99 \%$ confident that the samples are from two different populations (Cumming, Fidler, \& Vaux, 2007). The MANOVA method may also be used to compare multiple fields with each other, and the three methods are expected to produce similar results (Keselman et al., 1998).

Oneway analysis using JMP Pro 13 generates "mean diamonds", which illustrate both the sample mean and confidence interval. The top and bottom of each diamond represent the $(1-\alpha) \times 100$ confidence interval for each group. The confidence interval computation assumes that the variances are equal across observations, and as such the height of each diamond is proportional to the reciprocal of the square root of the number of observations within the group. The mean line across the middle of each diamond represents the group mean, while the overlap marks appear as lines above and below the group mean. In instances where groups have equal sample sizes, these overlapping marks indicate that the two group means are not significantly different at the given confidence level. Where the mean in one diamond is between the overlap marks of another diamond, this indicates that these two groups are not significantly different at that confidence level (SAS Institute Inc., 1989-2007).

\section{Materials and Methods}

\subsection{Sample Collection}

The field work was conducted on the KAPRORET tea estate in Kericho, Kenya, with latitude: $0^{\circ} 22^{\prime} 3.86^{\prime \prime} \mathrm{N}$ and longitude: $35^{\circ} 16^{\prime} 59.30^{\prime \prime} \mathrm{E}$, during January and February of 2017. Based on historical in-filling records, two good fields, fields $12 \mathrm{~A}$ and 12B, with fewer in-fillings due-to-deaths-by-drought (planted from a good batch of seeds) and two poor fields, 13A and 13B, with higher in-fillings due-to-deaths-by-drought (planted from a poor batch of seeds) were selected. These fields were approximately 1200 meters apart. The fields were planted from different batches of seeds obtained from Assam, in 1954 and 1956 respectively. They were in the same prune year, receiving the same fertiliser regimen, under rainfed conditions. The longitude and latitude coordinates for field $12 \mathrm{~A}$ and $12 \mathrm{~B}$ are $35^{\circ} 14.75^{\prime} \mathrm{E}, 0^{\circ} 26^{\prime} \mathrm{S}$ and for field $13 \mathrm{~A}$ and $13 \mathrm{~B}$ are $35^{\circ} 15.05^{\prime} \mathrm{E}, 0^{\circ} 26.6^{\prime} \mathrm{S}$ and at an altitude of $2180 \mathrm{~m}$ above mean sea level. Samples were collected using a "point-intercept within a quadrat method", in which a $100 \times 100 \mathrm{~m}$ quadrat was set up in the middle of each field. The starting point of the sampling was noted as point $(0,0)$. Each quadrat consisted of intersecting lines along ten meter by ten meter pre-determined points on the transect line. This essentially gave ten rows along the "x-axis" and ten rows along the " $y$-axis". At each intersecting point, three shoots of two leaves and a bud were harvested from each tree and placed in zip-lock plastic bags. A total of 400 samples were collected, 100 from each of the two good and two poor fields. Following sample collection, the leaves were transported to the Tea Research Institute laboratory, with the zip-locked plastic bags placed in an insulated box on ice. The samples were then subjected to the SWAPDT method as discussed in Nyarukowa et al. (2016) to determine the relative water content of the leaves from each field. The SWAPDT method is an inexpensive and practical method developed for the prediction of DT tea cultivars. The rate of relative water content (RWC) loss between the DT and DS cultivars was evaluated by immersing three shoots with two leaves and a bud from a single bush under investigation in $20 \mathrm{ml}$ of distilled water at room temperature and weighed after 24 hours. These turgid leaves were then blot dried and weighed before being oven dried at $37{ }^{\circ} \mathrm{C}$ and weighed after five hours when their RWC is between $40-80 \%$. The leaves were again placed in water for 24 hours, weighed after and oven dried at $105{ }^{\circ} \mathrm{C}$ for 24 hours to obtain each leaf's dry weight. 


\subsection{Statistical Analysis}

The data collected on the four fields, were tested for spatial homogeneity. In the case of field 13B, spatial dependence was detected, as provided by Bivand et al. (2011). A spatial simultaneous autoregressive error model was fitted to the data of field 13B, to predict and consequently remove the spatial signal. This was done using the "spdep" package. Using the one-sided Mann-Whitney test, it was established that Fields 12A and 12B, and 13A and 13B were respectively similar, but that the two groups differ significantly from one another. A Monte-Carlo permutation test approach was then employed, using the p-value of the one-sided Mann-Whitney test as test statistic, applied to comparisons between Fields 12A and 13A, 12A and 13B, 12B and 13A, and 12B and 13B. One thousand repetitions of this test were performed at decreasing sample sizes, to construct an empirical distribution of the p-value for each sample size. The empirical quantile at which 0.05 and 0.01 was observed was recorded. As the sample sizes decreased, the stability of the Mann-Whitney test decreased, as could be seen by the quantiles which usually lead to the rejection of the null hypothesis now moving away from the right-tail of the empirical distribution. The minimum required sample size was set at the level just before the empirical quantile dropped below the required significance level. This test procedure was also repeated while controlling for the mean difference in fields, and by combining the data for Fields $12 \mathrm{~A}$ and 12B, and 13A and 13B into Fields 12 and 13, respectively.

Power curves for the tea data set were calculated using the package "pwr". In Figure 4, four effect sizes have been defined, which correspond to a difference of $3 \%, 5 \%, 10 \%$ and $15 \%$. The $3 \%$ and $5 \%$ represent small effect sizes, with the $10 \%$ representing a medium effect size and the $15 \%$ a large effect size. The difference found in the pilot data set $(35 \%)$ would be considered a gigantic effect size. The curves were created for the different amount of fields included in the experiment; these curves serve to determine a suitable number of replicates required for each field for example:

When the desired power is .90 and the smallest relevant effect size is $5 \%$ and if 100 fields are used in the experiment, the minimum number of necessary replicates is estimated at approximately 22 . When 50 fields are used, the minimum number of replicates would be about 33. The sample size calculations are done after eliminating any other "field effects" i.e. using oneway ANOVA. Where "field effects" are not eliminated, a mixed model approach is employed by adding random indicators for fields to the model. This addition results in an increase in the power. Oneway ANOVA was used to compare the two good and the two poor fields and compute their means, standard deviations, and the Student's t-test, and contour plots of each field were prepared by JMP Pro (ver 13). Excel was used to calculate the SEM, from the same Standard Deviation (SD) at different sample sizes and to obtain the equation of the curve.

\section{Results}

\section{$4.1 \%$ RWC Drought Score Contour Plots Based on SWAPDT Method}

The contour plots for the four fields are shown in Figure 2. These are indicative of the $\%$ RWC profiles, which were flat; this eliminates any possible bias due to underground rivers or rocky outcrops. The ANOVA comparison between the two good and the two poor fields shows clear differences, with the mean \% RWC of 72.2 for the two good fields, and 35.0 for the two poor fields ( $<<0.0001)$. The SD ranged from 13.3-20 units, indicating a large variation within each field. This is supported by the large coefficient of variation (CV) values, shown in Figure 3. 

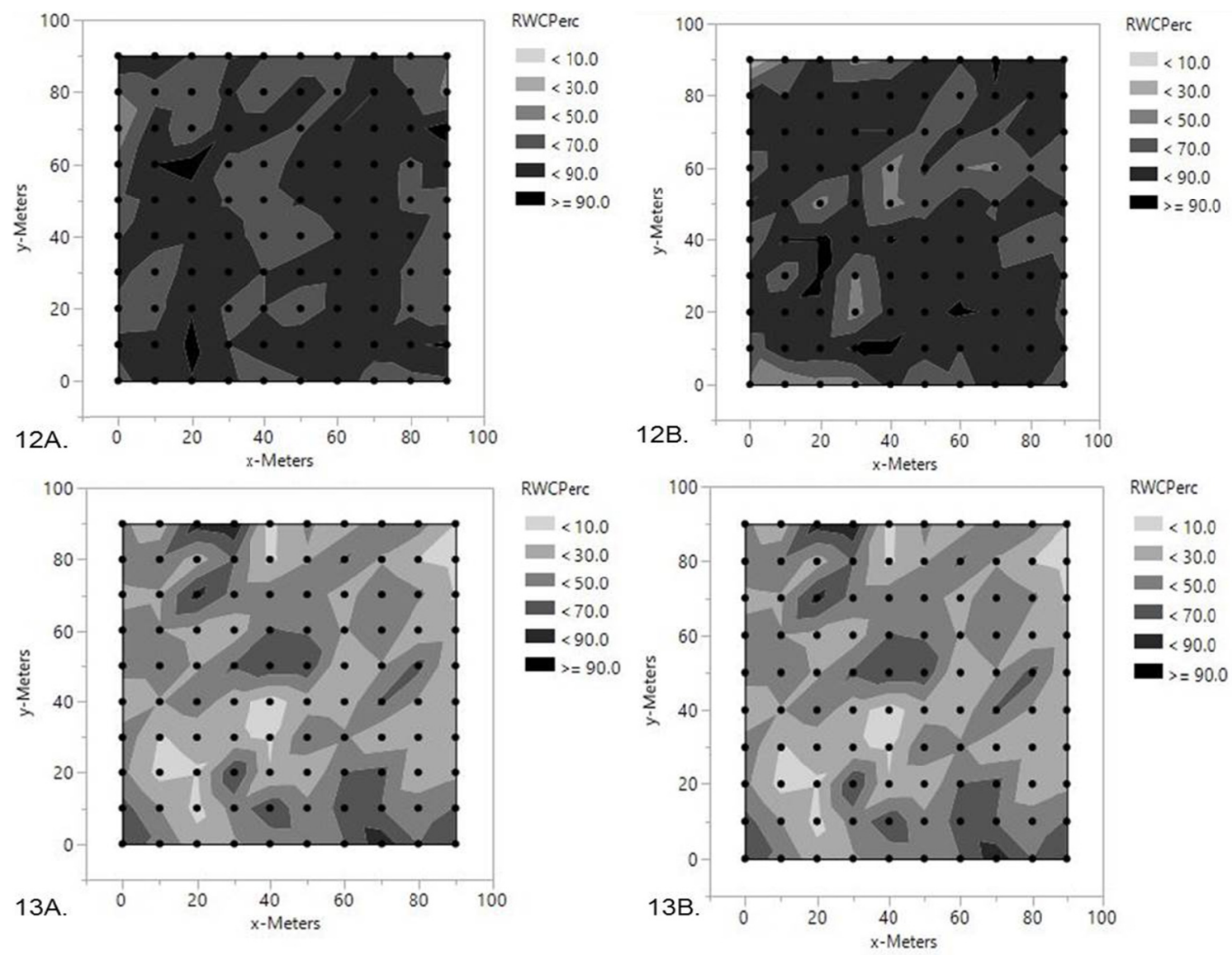

Figure 2. \% RWC drought score contour plots based on SWAPDT method for the two good fields (12A and 12B) and the two bad fields (13A and 13B)
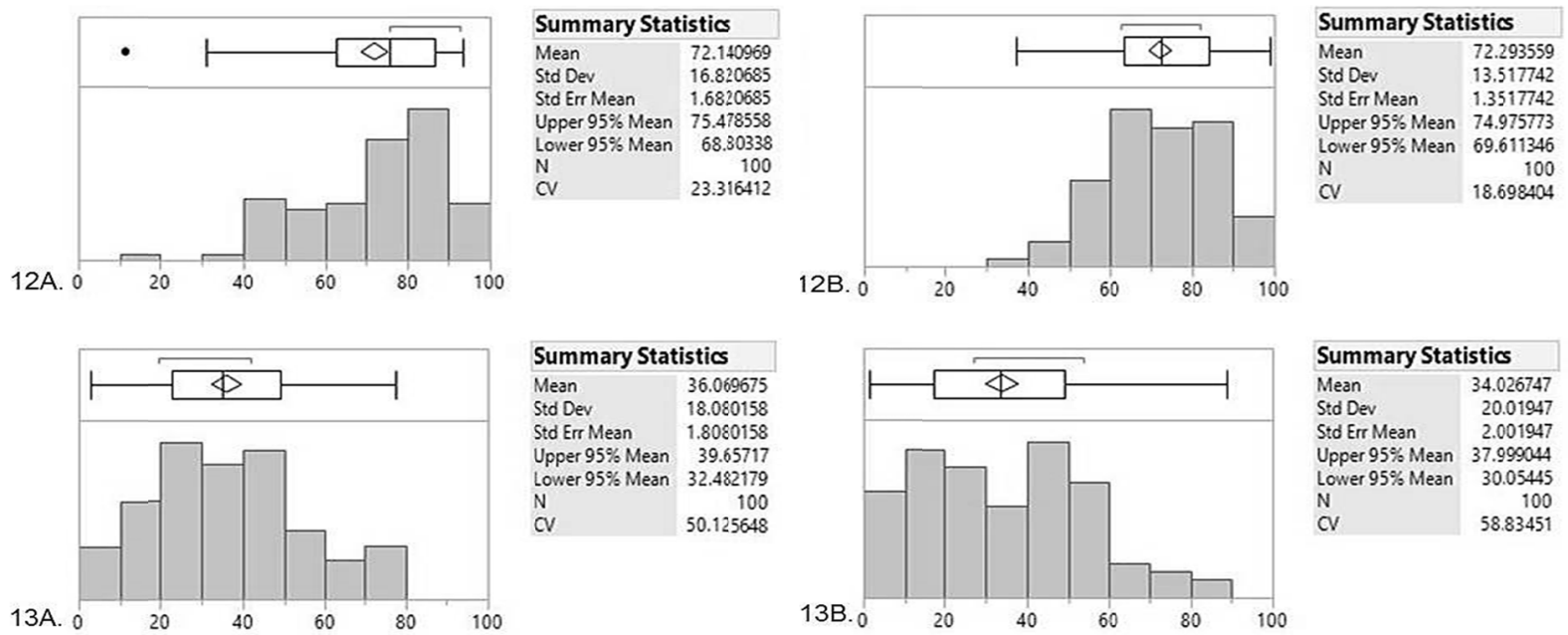

Figure 3. Mean distribution curves for the two good fields (12A and 12B) and the two bad fields (13A and 13B). The plots show the mean, std dev, SEM, sample size and the CV for each field

By approximation, the difference between the two means was expected to be statistically significant provided the difference of the means $>$ the sum of their SEMs or if Mean $1-$ Mean2 $>$ SEM1 + SEM2. The data for the two good fields were pooled and annotated as Good 1 and Good 2, while that of the poor fields was annotated as 
Poor 1 and Poor 2. The SEM for different sample sizes $(n=100,50,25,20,15,10$ and 5) for Good 1 and Good 2, and Poor 1 and Poor 2 pools were calculated using the equation shown in Figure 4.

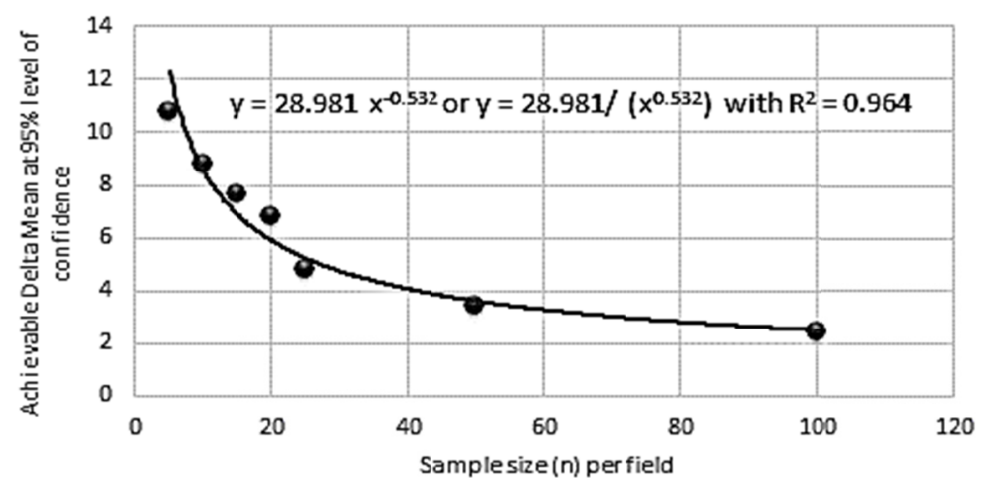

Figure 4. Plot shows the initially postulated number of samples per field deemed practical versus the actual statistically obtained sample number at the $95 \%$ level of confidence

It is important to tea estate management to know the sample size for two fields whose means are close to each other. Figure 4 shows the required numbers of samples per field versus the delta mean at the $95 \%$ level of confidence. The figure shows that if the delta mean is $8 \%$, the corresponding sample size is approximately 12 . Using the SWAPDT method and a sample size of 20, it is possible to distinguish between fields with a delta mean of $6 \%$. The collection of these 20 samples should be in the middle of the field to dispel any possibility of edge effects, and about 10 meters apart within rows and 10 meters apart between rows. Figure 5 below shows the Oneway analysis ANOVA results of the \% RWC of the two good and two bad fields. 


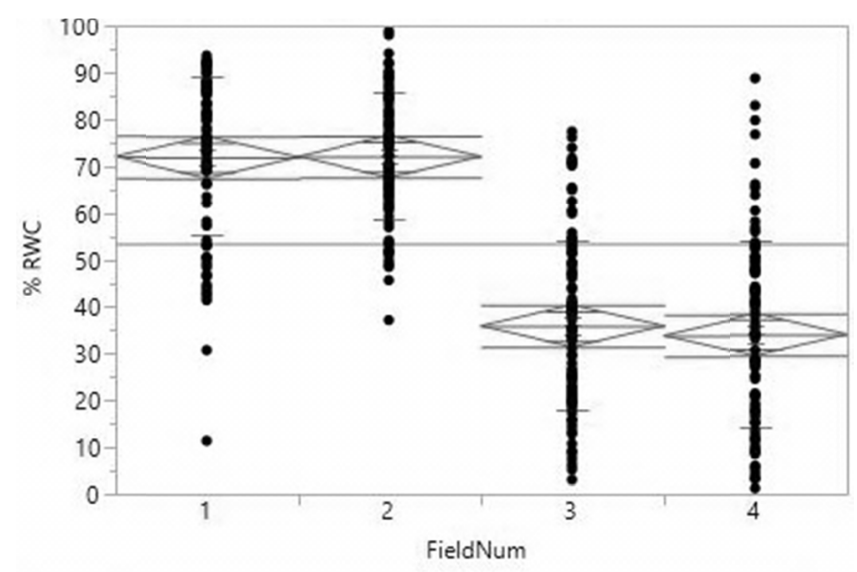

A.
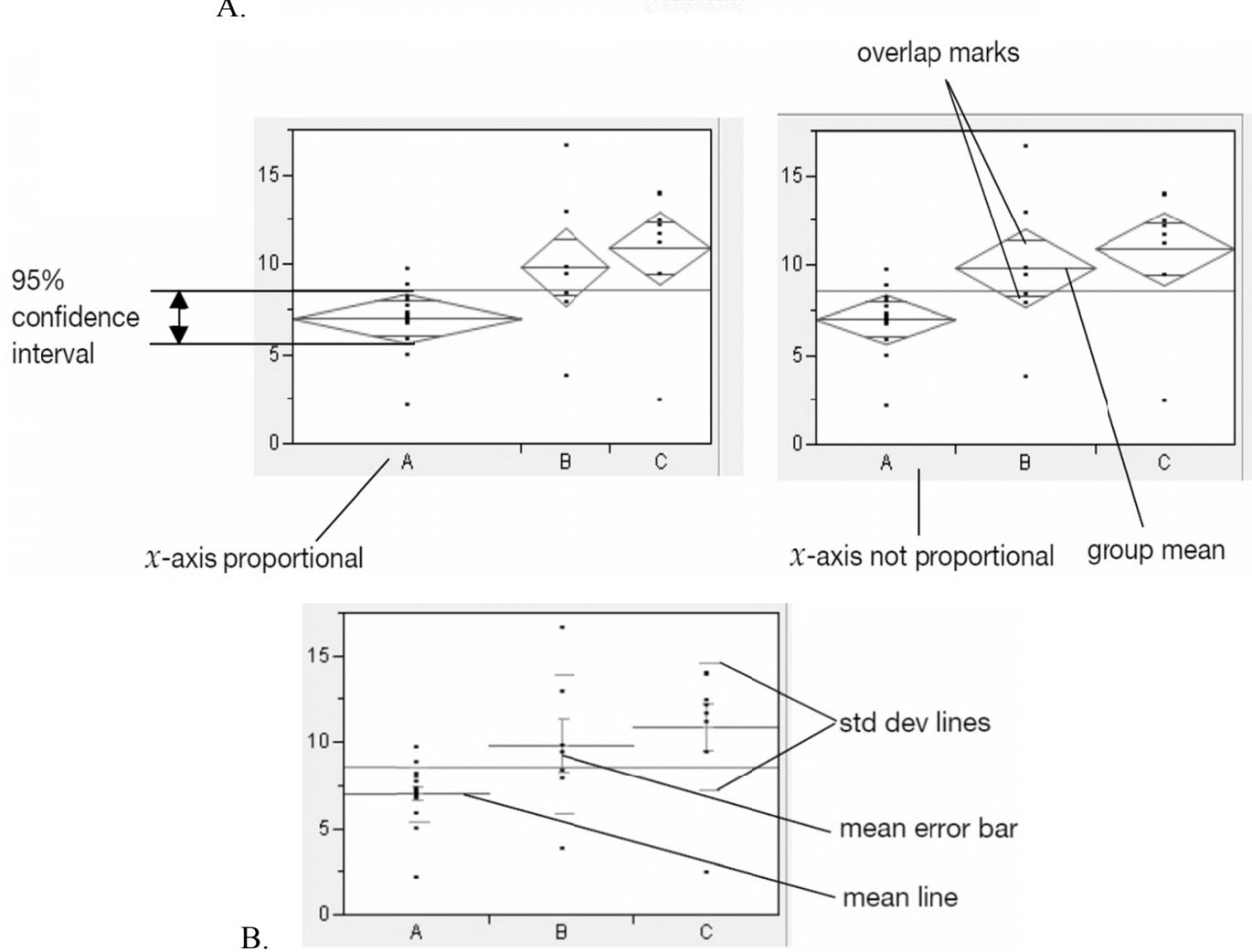

Figure 5. (A). Oneway analysis of the \% RWC against the two good and two bad fields. Oneway ANOVA was used to calculate the means. (B). Examples of Mean Diamonds and X-Axis Proportional Options. Mean Lines,

Mean Error Bars, and Std Dev Lines. (SAS Institute Inc., 1989-2007)

Power curves for the data set were also plotted in Figure 6, showing the four effect sizes which correspond with a difference of $3 \%, 5 \%, 10 \%$ and $15 \%$, as described above in the methods. 

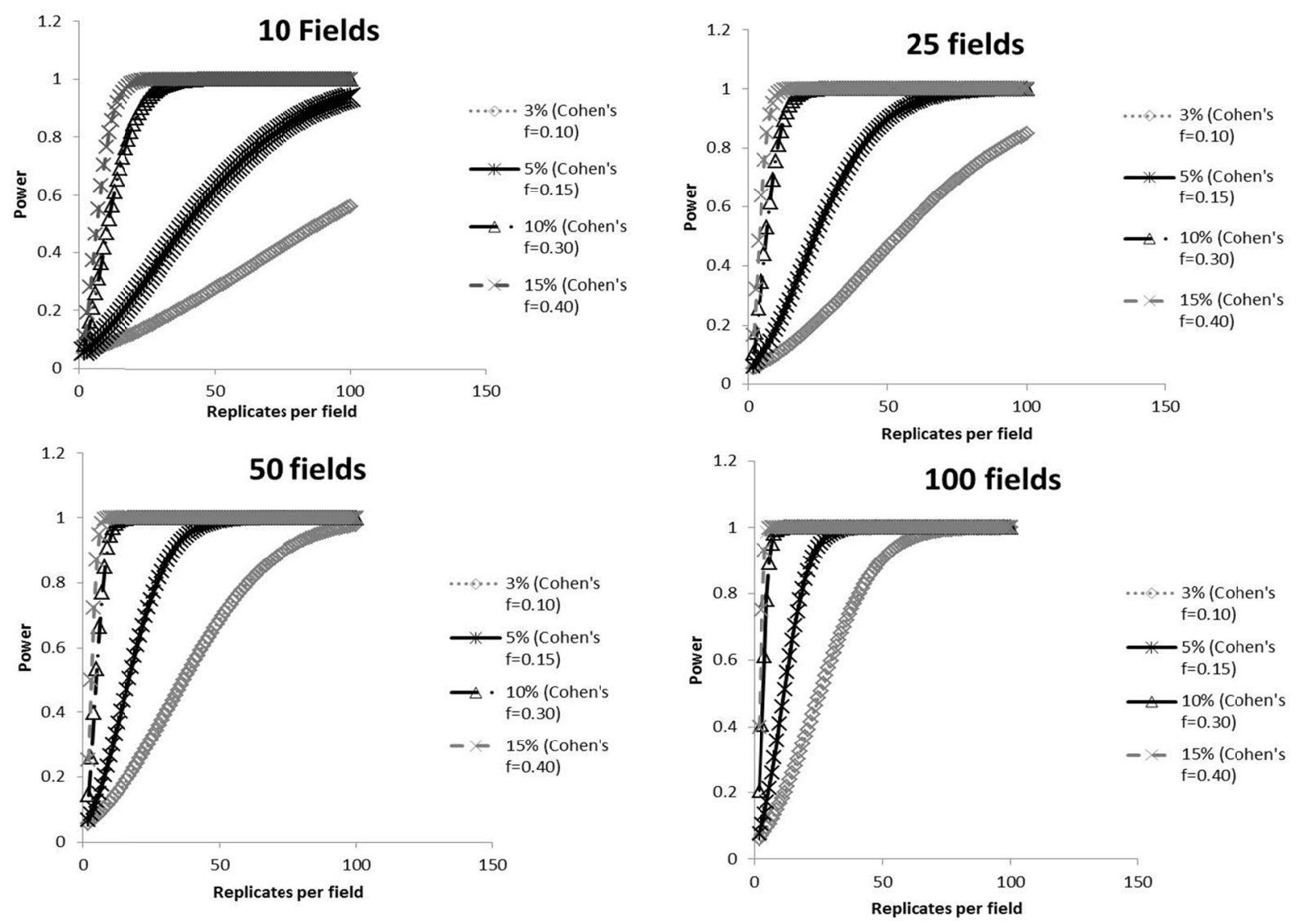

Figure 6. Power curves for the data set were plotted, showing four effect sizes which correspond with a difference of $3 \%, 5 \%, 10 \%$ and $15 \%$

\section{Discussion and Conclusion}

The RWC contour maps are almost flat, suggesting that there are no geological features that affect SWAPDT method scores, in these fields.

The results presented in the ANOVA showed that the SWAPDT method distinguishes good fields from poor fields. These findings correspond with the historical records of in-filling that were available for these fields. This finding suggests that where historical in-filling records are not available, the SWAPT method may be used to prioritize fields for replanting. These results also show that the SWAPDT method developed on tea cultivars from the TRFCA in Malawi can be applied to the seedling tea fields in Kenya, suggesting that the SWAPDT method may apply to other tea growing regions of the world. The sample size of 20 tea trees per field is sufficient to distinguish between fields that vary by $6 \%$ or more in their mean SWAPDT score. This sample size will need to be increased if the CV within a field is greater than $60 \%$, i.e. if fields were planted with a more heterogeneous source of seeds than used in the four fields reported here.

\section{References}

Avelino, J., Willocquet, L., \& Savary, S. (2004). Effects of crop management patterns on coffee rust epidemics. Plant Pathology, 53(5), 541-547. https://doi.org/10.1111/j.1365-3059.2004.01067.x

Besag, J., \& Diggle, P. J. (1977). Simple Monte Carlo tests for spatial pattern. Applied statistics (pp. 327-333). https://doi.org/10.2307/2346974

Bivand, R. S., Hauke, J., \& Kossowski, T. (2013). Computing the Jacobian in Gaussian spatial autoregressive models: An illustrated comparison of available methods. Geographical Analysis, 45(2), 150-179. https://doi.org/10.1111/gean.12008

Bivand, R., Anselin, L., Berke, O., Bernat, A., Carvalho, M., Chun, Y., ... Lewin-Koh, N. (2011). SPDEP: Spatial dependence: Weighting schemes, statistics and models. CRAN. Retrieved from https://cran.r-project. org/web/packages/spdep/index.html 
Cumming, G., Fidler, F., \& Vaux, D. L. (2007). Error bars in experimental biology. The Journal of Cell Biology, 177(1), 7-11. https://doi.org/10.1083/jcb.200611141

DaMatta, F. M. (2004). Ecophysiological constraints on the production of shaded and unshaded coffee: a review. Field Crops Research, 86(2), 99-114. https://doi.org/10.1016/j.fcr.2003.09.001

Dupont, W. D., \& Plummer, W. D. (1990). Power and sample size calculations: A review and computer program. Controlled Clinical Trials, 11(2), 116-128. https://doi.org/10.1016/0197-2456(90)90005-M

Ellis, R., \& Nyirenda, H. E. (1995). A successful plant improvement programme on tea (Camellia sinensis). Experimental Agriculture, 31(3), 307-323. https://doi.org/10.1017/S0014479700025485

FAO (Food and Agricultural Organisation). (2015). Retrieved from http://www.fao.org

Kelejian, H. H., \& Prucha, I. R. (2010). Specification and estimation of spatial autoregressive models with autoregressive and heteroskedastic disturbances. Journal of Econometrics, 157(1), 53-67. https://doi.org/ 10.1016/j.jeconom.2009.10.025

Kelsey, J. L., Thompson, W. D., Evans, A. S., Faden, R. R., Beauchamp, T. L., King, N. M. P., ... Hermann, B. P. (1986). Observational Epidemiologic Studies.

Keselman, H. J., Huberty, C. J., Lix, L. M., Olejnik, S., Cribbie, R. A., Donahue, B., ... Levin, J. R. (1998). Statistical practices of educational researchers: An analysis of their ANOVA, MANOVA, and ANCOVA analyses. Review of Educational Research, 68(3), 350-386. https://doi.org/10.3102/00346543068003350

Mei, C. L., He, S. Y., \& Fang, K. T. (2004). A note on the mixed geographically weighted regression model. Journal of Regional Science, 44(1), 143-157. https://doi.org/10.1111/j.1085-9489.2004.00331.x

Murakami, T., Nakamura, J., Matsuda, H., \& Yoshikawa, M. (1999). Bioactive saponins and glycosides. XV. Saponin constituents with gastroprotective effect from the seeds of tea plant, Camellia sinensis L. var. assamica Pierre, cultivated in Sri Lanka: Structures of assamsaponins A, B, C, D, and E. Chemical and Pharmaceutical Bulletin, 47(12), 1759-1764. https://doi.org/10.1248/cpb.47.1759

Nyarukowa, C., Koech, R., Loots, T., \& Apostolides, Z. (2016). SWAPDT: A method for Short-time Withering Assessment of Probability for Drought Tolerance in Camellia sinensis validated by targeted metabolomics. Journal of Plant Physiology, 198, 39-48. https://doi.org/10.1016/j.jplph.2016.04.004

Ord, J. K., \& Getis, A. (1995). Local spatial autocorrelation statistics: Distributional issues and an application. Geographical Analysis, 27(4), 286-306. https://doi.org/10.1111/j.1538-4632.1995.tb00912.x

Preedy, V. R. (2012). Tea in health and disease prevention. Academic Press.

R Core Team. (2016). R: A language and environment for statistical computing. R Foundation for Statistical Computing, Vienna, Austria. Retrieved from https://www.R-project.org

SAS Institute Inc. (1989-2007). JMP ${ }^{\circledR}$ (Version 13). SAS Institute Inc., Cary, NC.

Savary, S., Willocquet, L., Elazegui, F. A., Teng, P. S., Van Du, P., Zhu, D., ... Srivastava, R. K. (2000). Rice pest constraints in tropical Asia: Characterization of injury profiles in relation to production situations. Plant Disease, 84(3), 341-356. https://doi.org/10.1094/PDIS.2000.84.3.357

Willson, K. C., \& Clifford, M. N. (2012). Tea: Cultivation to consumption. Springer Science \& Business Media.

\section{Copyrights}

Copyright for this article is retained by the author(s), with first publication rights granted to the journal.

This is an open-access article distributed under the terms and conditions of the Creative Commons Attribution license (http://creativecommons.org/licenses/by/4.0/). 